\title{
Digital appendix 6. 100-topic model keywords
}

Although based on a 100-topic model the table is comprised of sixty topics because it excludes those that are predominantly comprised of OCR-errors: for instance, topic 0's keywords are "tile tihe thie thle tie hlie andl that site lihe withl anid tle tire lher anld felt antd hlier." I took this measure because, even if OCR error topics had no effect on decision tree outcomes, they introduced irrelevant information. The OCR errors that remained did not seem to have any effect for predictions of gender and nationality. However, their presence even at very small rates made it impossible to use the chapter's integrated method to investigate a key issue explored elsewhere in $A$ World of Fiction: the relationship between metropolitan and provincial newspaper publication. Because text files harvested from provincial newspapers were more likely to contain OCR errors, the decision tree used their presence in remaining topics to differentiate titles based on their place of publication: for instance, predicting provincial publication based on a title having more than $1.0001 \mathrm{E}$ of topic 15 . While an interesting effect, the results are not helpful in an interpretive sense, because they obscured other differences that might have existed between metropolitan and provincial newspapers and made it difficult to unpack the meaning of results: that is, whether they arose from OCR errors or indicated interpretable topics.

\begin{tabular}{|r|r|l|}
\hline $\begin{array}{l}\text { Topic } \\
\text { number }\end{array}$ & $\begin{array}{l}\text { Dirichlet } \\
\text { parameter }\end{array}$ & $\begin{array}{l}\text { Topic } \\
\text { keywords }\end{array}$ \\
\hline 1 & 0.05095 & $\begin{array}{l}\text { school boy boys artist master work teacher painter class paint tutor studio } \\
\text { painting pupil books pupils education sketch college }\end{array}$ \\
\hline 3 & 0.02575 & $\begin{array}{l}\text { train carriage platform railway ticket wur luggage passengers engine } \\
\text { compartment journey uv passenger travelling class wot express bag whistle }\end{array}$ \\
\hline 6 & 0.1428 & $\begin{array}{l}\text { town land country people years government men district city governor houses } \\
\text { trade class free capital place children inhabitants population }\end{array}$ \\
\hline 7 & 0.0693 & $\begin{array}{l}\text { officer general lieutenant soldier war officers men soldiers army sergeant } \\
\text { captain regiment service military colonel enemy troops uniform command }\end{array}$ \\
\hline 9 & 0.27593 & $\begin{array}{l}\text { mother dear girl sister child children papa poor father home girls lady pretty } \\
\text { tea room house daughter school brother }\end{array}$ \\
\hline
\end{tabular}




\begin{tabular}{|c|c|c|}
\hline 11 & 0.02784 & $\begin{array}{l}\text { gold tent mate mining miners miner diggings diggers shaft digger store creek } \\
\text { mates gully luck reef mines quartz golden }\end{array}$ \\
\hline 12 & 0.01059 & $\begin{array}{l}\text { gipsy monk duchesne quinlan gipsies overdown stanter montoith duggan } \\
\text { grandfather loda verschoyle helmstone osprey abbey limes murcott } \\
\text { peppercorn bossio }\end{array}$ \\
\hline 15 & 0.01071 & $\begin{array}{l}\text { pen shoulder owlet fry chunk jinkey vat star burtisland jethro lobo zo nigger } \\
\text { yorkey francey bolles plumper wid hermit }\end{array}$ \\
\hline 16 & 0.84396 & $\begin{array}{l}\text { boy head time round long half work hand give bit poor fellow night dog } \\
\text { called hands won morning boys }\end{array}$ \\
\hline 19 & 0.24294 & $\begin{array}{l}\text { flowers garden sweet trees sun golden lovely flower soft roses morning } \\
\text { beauty fair fairy birds leaves light music tree }\end{array}$ \\
\hline 22 & 0.03399 & $\begin{array}{l}\text { drink wine glass bottle landlord bar drunk drank whisky drinking liquor } \\
\text { glasses hotel beer bottles drunken drunkard champagne rum }\end{array}$ \\
\hline 23 & 0.01985 & $\begin{array}{l}\text { lad aye weel tae lass nae laddie dinna gang wad lassie sae tak granny wor } \\
\text { wee folk ony bairn }\end{array}$ \\
\hline 24 & 0.03416 & $\begin{array}{l}\text { count prince duke king princess countess royal majesty highness knight } \\
\text { chateau palace queen emperor chevalier court imperial duchess czar }\end{array}$ \\
\hline 26 & 0.07521 & $\begin{array}{l}\text { ring gold diamonds dress diamond jewels silk wear velvet satin rich silver } \\
\text { wore costly pearls marble dressed jewel box }\end{array}$ \\
\hline 27 & 0.65768 & $\begin{array}{l}\text { heart poor life child face mother man woman god eyes love dear wife long } \\
\text { tears father death time home }\end{array}$ \\
\hline 28 & 0.00943 & $\begin{array}{l}\text { fox hit scate bowler runs wicket secretary cricket wiggles gainsmere romer } \\
\text { chelps lacklyne match nuisance eleven worry stocks wickets }\end{array}$ \\
\hline 29 & 0.43128 & $\begin{array}{l}\text { man stranger gentleman friend returned lady woman moment hand strange } \\
\text { companion evidently door master entered turned appeared observed person }\end{array}$ \\
\hline 33 & 0.00902 & $\begin{array}{l}\text { baron baroness berrian decamp intendant tigg poiton layman seraphine } \\
\text { dinwiddie liimmergeier xrmak sudely jesuit flatten fiirst mornell dalway } \\
\text { duroo }\end{array}$ \\
\hline 34 & 0.67548 & $\begin{array}{l}\text { night moment light dead eyes hand heard voice death suddenly felt turned } \\
\text { stood feet life lay face strange terrible }\end{array}$ \\
\hline 35 & 0.02381 & $\begin{array}{l}\text { parliament political club president member politics members election vote } \\
\text { meeting government party liberal council governor committee secretary } \\
\text { elected assembly }\end{array}$ \\
\hline 37 & 0.02747 & $\begin{array}{l}\text { child baby woman nurse arms infant mercy wife mistress creature babies } \\
\text { cradle charge nursery sleeping basket madcap shawl born }\end{array}$ \\
\hline 38 & 0.01852 & $\begin{array}{l}\text { professor pit coal miner colliery miners manager mines pits prophet lemonpip } \\
\text { mining factory shaft working fessor engine sweyn regions }\end{array}$ \\
\hline 39 & 0.05281 & $\begin{array}{l}\text { rain storm wind snow night cold wet weather thunder lightning shelter clouds } \\
\text { warm falling frozen ice lantern stormy tempest }\end{array}$ \\
\hline 40 & 0.47291 & $\begin{array}{l}\text { river long road trees place sun ground dark feet hill lay stood distance light } \\
\text { hills path deep stream land }\end{array}$ \\
\hline 41 & 0.68031 & $\begin{array}{l}\text { man people woman life things time girl knew men talk poor place house } \\
\text { women suppose money looked sort years }\end{array}$ \\
\hline 45 & 0.40357 & $\begin{array}{l}\text { father mother son years daughter family brother man house wife boy home } \\
\text { died child death age married lived life }\end{array}$ \\
\hline 46 & 0.04021 & $\begin{array}{l}\text { smiles opens enters adds draws tells breaks walks passes puts hears brings } \\
\text { appears calls grows remarks throws repeats wears }\end{array}$ \\
\hline 47 & 0.22622 & $\begin{array}{l}\text { money man business pounds pay bank office lawyer clerk paid fortune give } \\
\text { sum work gold firm men gentleman cheque }\end{array}$ \\
\hline 48 & 0.33318 & $\begin{array}{l}\text { room door house night open hall light opened floor chair stood windows } \\
\text { entered woman rooms front place glass key }\end{array}$ \\
\hline 51 & 0.07495 & $\begin{array}{l}\text { uncle aunt cousin nephew niece dear auntie cousins relative ancle nieces } \\
\text { aunty guardian housekeeper wishes invitation relation nncle auut }\end{array}$ \\
\hline 52 & 0.72076 & $\begin{array}{l}\text { word give matter moment tone friend truth dear question room understand } \\
\text { hope reason poor wrong doubt hand brother felt }\end{array}$ \\
\hline 54 & 0.06807 & $\begin{array}{l}\text { lady lord countess lordship ladyship maid carriage woman servants title heir } \\
\text { valet noble nobleman court peer footman rank society }\end{array}$ \\
\hline 55 & 0.08725 & $\begin{array}{l}\text { doctor } \mathrm{dr} \text { patient nurse medical room case surgeon physician hospital sick } \\
\text { patients fever doctors medicine death health illness sleep }\end{array}$ \\
\hline
\end{tabular}




\begin{tabular}{|c|c|c|}
\hline 56 & 0.12453 & $\begin{array}{l}\text { king lord people priest god queen court church men country ancient blood } \\
\text { true called royal religion war prince catholic }\end{array}$ \\
\hline 57 & 1.08721 & $\begin{array}{l}\text { time night looked morning room home heard felt long evening knew turned } \\
\text { moment door house began coming half face }\end{array}$ \\
\hline 59 & 0.51472 & $\begin{array}{l}\text { man men life things human people nature book years point character fact } \\
\text { social sense natural books society general women }\end{array}$ \\
\hline 61 & 0.08555 & $\begin{array}{l}\text { squire farm farmer house country cottage hall road grange garden manor } \\
\text { fields land master county landlord wood hedge lodge }\end{array}$ \\
\hline 62 & 1.54492 & $\begin{array}{l}\text { time present fact place position long means state received order short large } \\
\text { friends manner small appearance called house case }\end{array}$ \\
\hline 63 & 0.22202 & $\begin{array}{l}\text { love girl heart marry loved lover dear wife marriage daughter married } \\
\text { happiness true life sweet give hand eyes lips }\end{array}$ \\
\hline 64 & 0.08903 & $\begin{array}{l}\text { ship captain sea board deck vessel boat sailor voyage mate men passengers } \\
\text { crew port steamer wind sailors man ships }\end{array}$ \\
\hline 65 & 0.02184 & $\begin{array}{l}\text { chief natives native canoe tribe islands island indians warriors savages war } \\
\text { chiefs canoes warrior savage pennant tribes jungle missionary }\end{array}$ \\
\hline 66 & 0.10813 & $\begin{array}{l}\text { horse horses ride road riding saddle whip rider stable animal race mounted } \\
\text { bridle pony gallop reins groom run pace }\end{array}$ \\
\hline 71 & 0.04747 & $\begin{array}{l}\text { goin afore reckon jest aint thar wot wasn folks gal wos feller bout kin tucker } \\
\text { mighty won guess agin }\end{array}$ \\
\hline 72 & 0.0868 & $\begin{array}{l}\text { murder case detective man prisoner evidence court witness death dead guilty } \\
\text { body judge jury committed murdered lawyer murderer general }\end{array}$ \\
\hline 73 & 0.01644 & $\begin{array}{l}\text { jew jews dwarf city jewish maiden allah rabbi sultan desert messiah prophet } \\
\text { hebrew palace turks gold sabbatai cumner arabs }\end{array}$ \\
\hline 74 & 0.03494 & $\begin{array}{l}\text { stage theatre play music actress audience manager night played performance } \\
\text { violin box curtain opera actor piece scene company applause }\end{array}$ \\
\hline 75 & 0.06085 & $\begin{array}{l}\text { wife husband married bride marriage wedding woman bridegroom bridal } \\
\text { ceremony church law carriage couple pair veil altar wives honeymoon }\end{array}$ \\
\hline 76 & 0.45671 & $\begin{array}{l}\text { lady ladies dear room friend dinner pretty party gentleman friends evening } \\
\text { fair handsome society girls dance fellow eyes pleasure }\end{array}$ \\
\hline 77 & 0.01248 & $\begin{array}{l}\text { temple towers watkin colpoys renton cinders jukes mayflower attorney } \\
\text { prophecy cruiklawn client esteemed inglis millet sharker lulgi dauby bouverie }\end{array}$ \\
\hline 78 & 0.13015 & $\begin{array}{l}\text { letter letters paper write written wrote writing post note papers office } \\
\text { envelope hand desk received dear pocket morning pen }\end{array}$ \\
\hline 80 & 0.06535 & $\begin{array}{l}\text { creek sheep cattle horses men verandah hut country man horse track township } \\
\text { blacks tree river squatter tea gum night }\end{array}$ \\
\hline 85 & 0.59453 & $\begin{array}{l}\text { life heart love nature beauty felt youth home feeling years power soul } \\
\text { pleasure long spirit thoughts full future loved }\end{array}$ \\
\hline 87 & 0.07882 & $\begin{array}{l}\text { boat sea shore beach island waves wind tide coast rocks boats land river } \\
\text { waters sand fish men fishing oars }\end{array}$ \\
\hline 89 & 0.0181 & $\begin{array}{l}\text { christmas ice psyche snow ghost skating cave bayley trumpet christ skates } \\
\text { haunted caves isaline christinas ghosts hamper lownd wombat }\end{array}$ \\
\hline 90 & 0.05848 & $\begin{array}{l}\text { church vicar parson parish clergyman curate service sermon congregation } \\
\text { cathedral god prayer chapel vicarage deacon churchyard reverend preacher } \\
\text { organ }\end{array}$ \\
\hline 91 & 1.03975 & $\begin{array}{l}\text { eyes face girl looked hand voice head man dark turned stood hands lips } \\
\text { moment smile half long woman low }\end{array}$ \\
\hline 94 & 0.13799 & $\begin{array}{l}\text { street city shop streets town cab crowd houses house man hotel carriage } \\
\text { driver place road door front landlady walked }\end{array}$ \\
\hline 96 & 0.04127 & $\begin{array}{l}\text { captain colonel soldier officer regiment military cigar army mount cope } \\
\text { merrydew service mess chaplain gallant prank bungalow honourable climate }\end{array}$ \\
\hline 97 & 0.01151 & $\begin{array}{l}\text { priory tempest vixen peacock oona plague hh pollio cocoa wingfield masque } \\
\text { moray barholme hj henslow hartfield pilbeam croke snag }\end{array}$ \\
\hline 98 & 0.24961 & $\begin{array}{l}\text { man men hand blood fight fellow blow life head knife pistol ground danger } \\
\text { party time body brave enemy killed }\end{array}$ \\
\hline
\end{tabular}

